\title{
On the Development of a Resident Monitoring System: Usability, Privacy and Security aspects
}

\author{
Pascal Bruegger, Adriana Wilde and Loïc Guibert
}

\begin{abstract}
Worldwide, the elderly have suffered disproportionately from the effects of the COVID-19 pandemic, both in terms of their prognosis once contracted the disease and in terms of the preventative measures required for this demographic, who are at much higher risk than the rest of the population. In the "new normal", the well-being of older adults (residing either in their own homes or in care homes) will be ideally monitored remotely. These measures would preserve the independence of individuals without compromising on their safety. In this paper we discuss aspects of the design and implementation of a resident monitoring system (RMS) with particular emphasis on overcoming the barriers for adoption among these populations, by addressing the aspects of usability, privacy and security at the core of the development of such a system. We discuss the current challenges of this research and future work on the RMS.
\end{abstract}

Index Terms-Resident monitoring, acceptability, smart homes, vulnerable groups, ethics.

\section{INTRODUCTION}

$\mathrm{T}$ He elderly population of the world has suffered disproportionately from the effects of the COVID-19 pandemic [1]. In addition to the physical and mental health challenges of this age group, they face extreme isolation, in the current times but also in the post-COVID-19 era. This isolation comes as a consequence of many governments having implemented measures for infection prevention and control (IPC) as recommended by the World Health Organisation [2]. Strict social distancing measures have been adopted, which are typically more severe for vulnerable groups such as the elderly and those living with certain disabilities. Therefore, the development of a resident monitoring system is more important than ever, as it would facilitate the independence of individuals without impacting negatively

- P. Bruegger and Loïc Guibert are with the School of Engineering and Architecture of Fribourg University of Applied Sciences Western Switzerland. Fribourg, Switzerland $\mathrm{CH}-1705$

E-mail: Pascal.Bruegger@hefr.ch,Loic.Guibert@hefr.ch

- A. Wilde is with the Centre for Health Technologies (CHT) in the Electronics and Computer Science at the University of Southampton, UK, SO17 1BJ and with the Department of Digital Technologies at the University of Winchester, UK, SO22 4NR. E-mail:agw106@soton.ac.uk, adriana.wilde@winchester.ac.uk

Manuscript received August 10, 2020 on their safety and well-being. Further, the benefits of the adoption of such a system would extend to their relatives and carers, as they could enjoy the peace of mind that remote monitoring would offer, when reliable and safe to use. For doctors, who may already suffer in their mental health due to fears of contracting COVID-19 themselves [3], it would be valuable to be able to monitor patients remotely too. Moreover, it is hard for an elderly person to decide whether to move into a nursing home after having led an independent life. This independence is replaced by a structured regime where activities, meals, and care are somewhat restricted. This independence is traded due to the need for regular medical care or supervision given the heightened risk of injuries and falls, or a diminished ability to perform activities of daily living (e.g. grooming, feeding and taking medication).

Unobtrusive monitoring of residents may support a perceived balance of independence, safety and well-being. An idealised system supporting semi-independent living is an ecosystem that includes sensors that can localise and detect residents' activities and collect physiological data, and send secure updates about their state to the most appropriate available person able to intervene, if needed. 
This paper is organised as follows: in Section 2 we look at previous work, leading into the description of our resident monitoring system in Section 3. Section 4 discusses an exemplar application of the system: fall detection. Section 5 covers specific measures addressing the privacy and security concerns which would prevent this system being adopted. Finally, current challenges, future directions and conclusions are discussed in Sections 6, 7 and 8.

\section{StATE OF THE ART}

Smart home technologies promise to help elderly adults and other vulnerable individuals maintain a good quality of life [4]. Such technologies can incorporate a variety of features allowing the use of sensor data both for activity recognition and timely detection of anomalies [5] which would offer valuable information for doctors and carers to act upon, to preserve the well-being of the patient.

Several academic and commercial projects are based on the idea of connecting the person to a response centre which answers when a panic button is pressed, and are designed for independent living. Notable examples are: IPI Singapore Elderly Monitoring System ${ }^{1}$, Philips Lifeline ${ }^{2}$, Medical Guardian ${ }^{3}$, and Rescue Alert ${ }^{4}$. Their main shortcoming however, is that the person is required to activate such a signal, making the technology obstrusive at best, and unusable in most cases.

Indeed, despite the development of several assistive systems tailored to independent living, the rates of adoption of health technologies is still very low among the elderly [6]. Further, there are ethical and acceptability challenges which may be contributing factors to such rates [7]. In particular, concerns regarding privacy and security, are very important to address for an increased adoption and therefore effective monitoring of the well-being of older adults living independently.

\section{Resident Monitoring System}

How best to monitor residents' well-being whilst supporting independence for the residents and

1. https://www.ipi-singapore.org

2. https://www.lifeline.philips.com

3. https://www.medicalguardian.com

4. https://www.rescuealert.com/

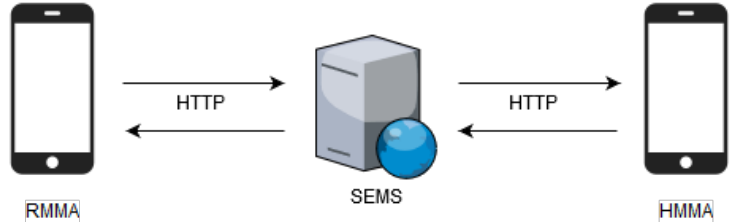

Fig. 1. Resident Monitoring System (RMS) high-level architecture. Two mobile applications (RMMA and HMMA) are connected through the SEMS server, via HTTP links. RMMA sends physiological data of the residents to HMMA, and raises an alarm on certain health events (see Section 3 ).

also relieving some of the pressure on healthcare providers without sacrificing the safety of their users? An idealised monitoring solution should be a usable, secure and trusted ecosystem that includes reliable sensors, robust telecommunication capabilities and usable user interfaces. Sensors can localise and detect residents' activities and collect physiological data, a way of sending regular updates about the situation of the residents they take care of to medical staff and a central monitoring system for both residents and medical staff and a logic to to support decision-making about the most appropriate available person to intervene in case of problems with a resident.

In this paper we present our exploration and development of a human-centric solutions that use new technologies in the context of a nursing home. Namely, the creation of an intelligent environment that monitors, in a non intrusive way, the situation of residents in a broad sense, meaning their physiological state, level of activity and their location. To that end, our team built an ecosystem of three prototype applications ${ }^{5}$, illustrated in Figure 1. In what follows, we proceed to describe these applications. Firstly, there is the Resident Monitoring Mobile App (RMMA), which collects and manages six main types of information: the resident's identity, heart rate, activity, location, fall detection and medication reminder. This application is able to generate alarms when abnormal behaviour occurs (e.g. excessive heart rate or suspicious activity). All information, including alarms, is sent to a server that is part of the second project: Smart Environment Monitoring System (SEMS) and SEMS-UI (illustrated in Figures

5. Early prototypes were implemented by students at the School of Engineering and Architecture of Fribourg (HEIA-FR). 


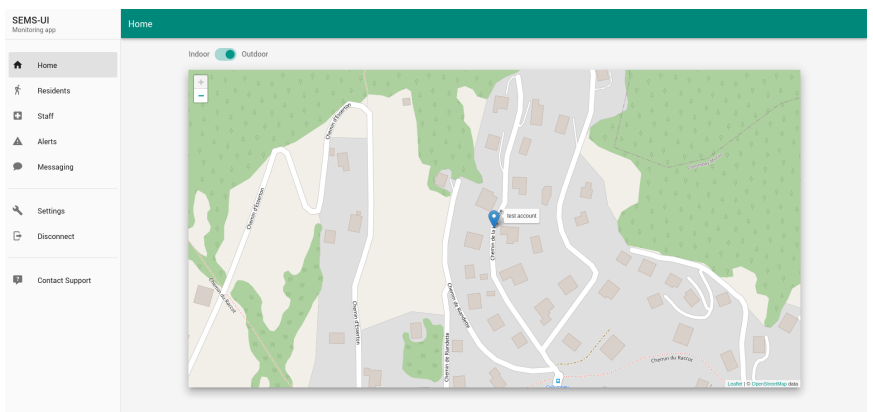

Fig. 2. SEMS-UI, web interface used by the medical staff to locate the resident and the medical staff (See Section 3).

2 and 3). The SEMS server has been developed using the Spring Java framework, with its Boot derivative. The purpose of the server is to centralise the information about the residents and to manage the alarms coming from the resident's application. The simple decision-making process makes it possible to choose the right person for an intervention with the resident: either the nearest person or the person most competent to handle the problem. The third project consists of a mobile application for medical staff: Health Monitoring Mobile App (HMMA). This application allows staff to get data about residents and receive alarms from residents whose medical person is responsible. This is done through the server. Among other things, it allows the server to be informed about the availability of medical personnel. The two mobile applications can communicate with each other, through the Application Programming Interface (API) of the SEMS server and transmit messages. Together, the three projects form a complete ecosystem that creates an intelligent environment which takes into account the activities.

One of the forces of the RMS ecosystem is its ease of adoption, as much in the used hardware and its installation. All the physical devices, sensors and its overall architecture do not require specific material nor proprietary technologies. Its development has been conducted with openness in mind. Regarding the needed adaptations in the buildings of the monitored ecosystem, we only need to place beacons in strategic parts of the rooms. Those beacons permit a precise indoor location tracking of both residents and the medical staff.

\section{FALL DETECTION FOR ELDERLY PEOPLE}

In the context of the SEMS project, we integrated the result of a master thesis on fall detection for elderly [8]. Falls are one of the leading causes of death among the elderly [9]. Every year, $28 \%$ to $35 \%$ of elderly people experience (at least) a fall and the likelihood of such an event and the severity of the outcomes increase with age [10]. Falls can have severe physical, psychological and even social consequences. They can also heavily affect the independent quality of living. They can result in bruises and swellings, as well as fractures and traumas [11]. A significant risk is the long-lie. This happens when an elderly person remains on the ground for a long duration without being able to call for help. It is associated with death within the next few months following the accident [12]. It also affects the elderly's self-confidence who may develop the fear of falling syndrome. It leads to anxiety when performing Activities of Daily Living (ADLs) and can lead to subsequent falls [9]. Therefore, the elderly must continuously be monitored to ensure their safety. Families organize visits but these can be inconvenient and even insufficient. Hiring caregivers or moving into nursing homes are sometimes not affordable options. Recent progresses in technology have enabled the development of Assisted-Living Systems (ALSs) [13]. They can assist the elderly and provide a safer environment through constant monitoring while relieving caregivers' workload. Therefore, we have added a Machine-Learningbased component for fall detection [8] in the SEMS server to complete the monitoring system. In order to find the best solution, two major subsets of $A r$ tificial Intelligence systems have been evaluated for this context: Machine-Learning and Deep-Learning. Various models have been assessed, and the bests results were obtained with the Random Forest (RF) and Gradient Boosting (GB) algorithms. However, ALSs create other challenges such as privacy concerns and acceptability issues that need to be addressed [14].

\section{Privacy and Security concerns}

Important aspects of the project include the humancomputer interaction, privacy and security considerations, which in this case are strongly coupled. For the applications to be accepted by residents and 


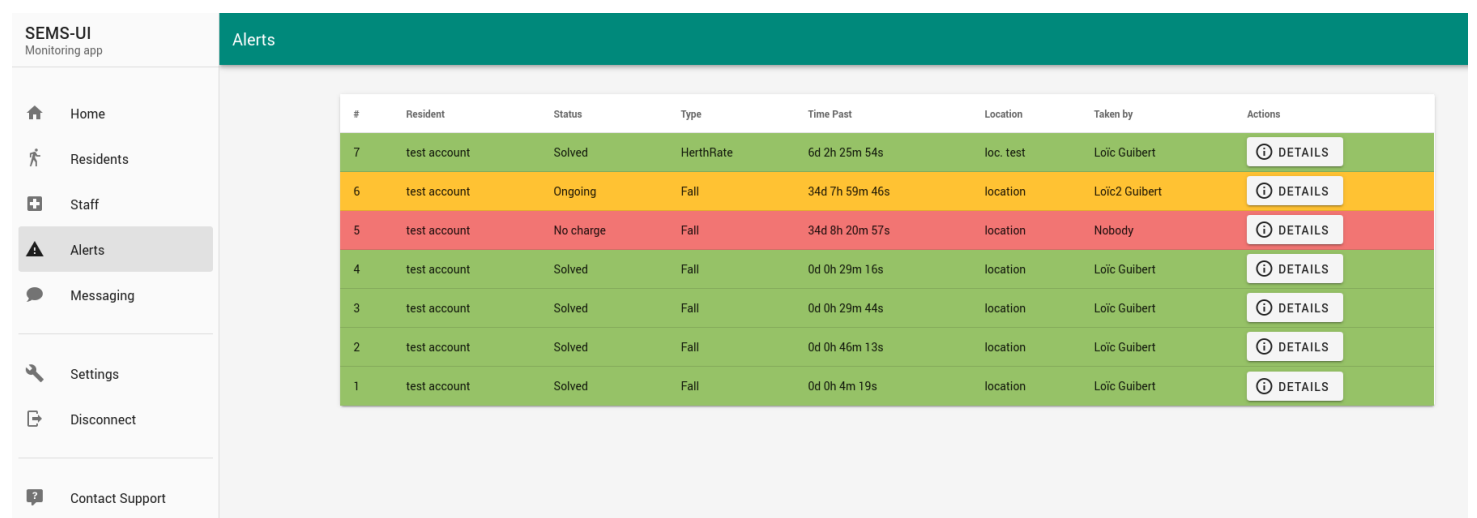

Fig. 3. SEMS-UI: visualising the raised alerts about residents, including its type, status, elapsed time, and location.

medical staff, they must meet specific needs, be easy to use, be non-intrusive, and assure users that their privacy is respected and that their data, which in the case of health data can be very sensitive, is secure. The monitoring systems must be adapted to residents' physical (sight, hearing, touch) and cognitive (memory, literacy, etc.) conditions. Finding the most appropriate sensors and devices to capture the physiological data is a key component of the project. For example, while chest belts are a good solution to monitor heart rate for athletes, who usually only wear them for a short time, they may not be ideal for the elderly since they would have to constantly wear them and would likely find them uncomfortable over time. A connected watch or a bracelet would be a possible, and probably more suitable solution in this case.

We developed a smartwatch solution called Smartwatch Resident Monitoring App (SRMA, shown in Figure 4) using an Android-based smartwatch. Currently, a light Bluetooth-connected wristband including a blood pressure monitor is studied.

Also the user interface must be easy to understand and use for a variety of users with different cognitive abilities, and may need to be adaptable as abilities degrade over time. As regards the mobile application for the medical personnel, it also needs to be simple, easy to use and adapted to their needs, but for different reasons. The medical personnel would have limited time since they have a heavy workload involving a large number of patients, so they need to take decisions quickly, managing messages from different sources efficiently, and with little margin for error. The design of the interface
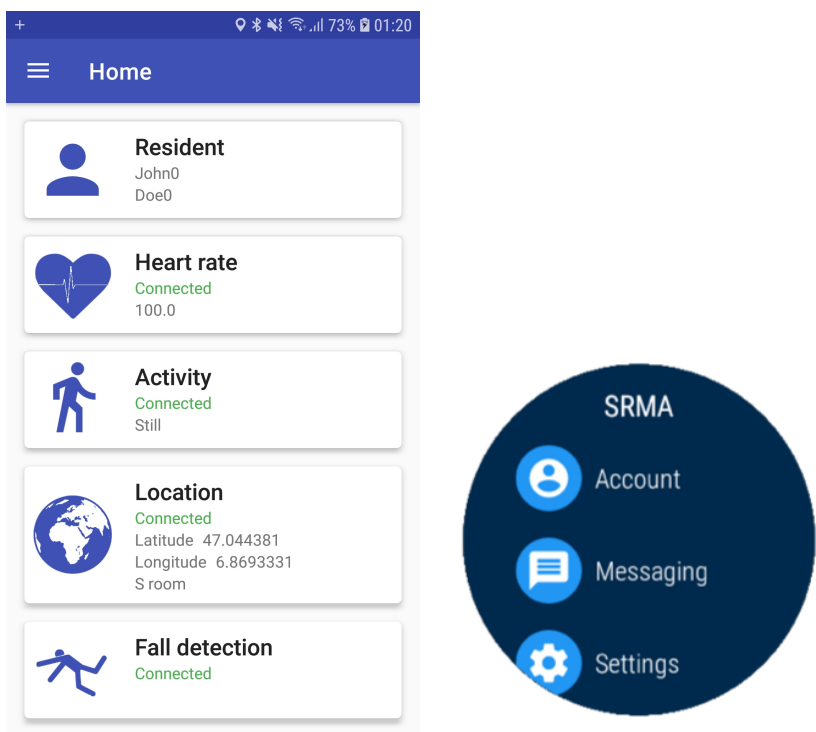

Fig. 4. Main user interfaces of the Resident Monitoring Mobile App (RMMA, to the left) and Smartwatch Resident Monitoring App (SRMA, to the right). The goal of the design of the interfaces is to be as simple and as usable as possible.

therefore takes all of these factors into account. Any applications that involve health data and/or person tracking must imperatively address questions of privacy and security. Because of the types of medical data being collected, who has access to that data needs to be decided judiciously for ethical reasons, and transfer of that data is secured by encryption protocols (HTTPS, TLS). Other mechanisms must be implemented for enforcing security: all the RMS users sessions are automatically linked to auto-generated tokens and are limited in time. The permissions system must also be improved for a 
better management of users' access rights. Knowing where residents and staff are, and potentially what they are doing (derived by combining data from different sources) leads to potential invasions of privacy. Two aspects are investigated now. The first is what level of this type of invasion of privacy might be acceptable, given the trade-off between privacy and access to potentially life-saving assistance. The other is what types of Privacy Enhancing Technologies might be applicable in this case that would allow maximum preservation of privacy without undermining the utility of the application. A survey to investigate the two aspects is being conducted in various nursing homes in Switzerland with the participation of elderly residents and medical staff.

\section{Current challenges}

The acceptability of such system is probably one of the main challenges. The result of the survey will be crucial for the research. Depending of the perception of the ecosystem by the elderly and the medical staff, we will need to adapt our approach.

Another challenge we are working on is the notification system. If a problem is detected by the resident application (e.g. Fall or high heart rate), an alarm is send to the SEMS server and then send to the medical staff application. For that, we have recently replaced the push notification and authentication provided by Google Firebase ${ }^{6}$ (Figures 5) with a system based on Socket.IO and our server MAAS (Messaging And Alert System) (Figures 6). We took the decision to undergo these changes because in the previous setup, medical data were in transit via Firebase (and Google servers). This was not regarded as secure and it is important to comply with GDPR and other European directives for the management of health data in the context of this research. We not have a complete self-sufficient system.

A related research question that we are currently exploring is regarding the decision-making process on who should be alerted in case of an event that requires intervention. This is a complex process with several variables involved, including: the severity of the event, the location of the resident, the medical staff availability, competencies and their

6. https:/ / firebase.google.com/products

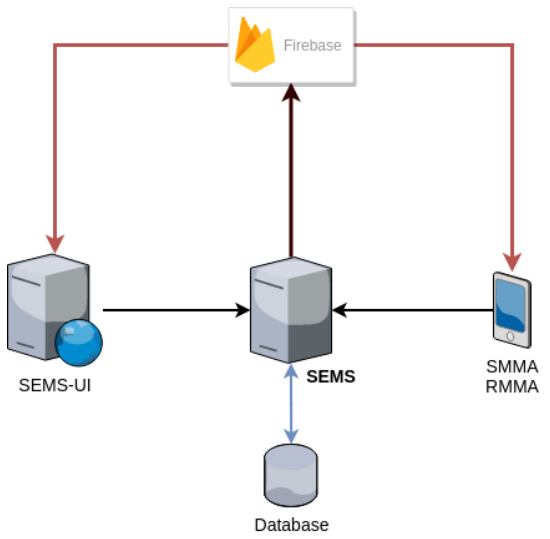

Fig. 5. Earlier prototype: the connection SEMS-RMMA-HMMA used Firebase for push notification and authentication.

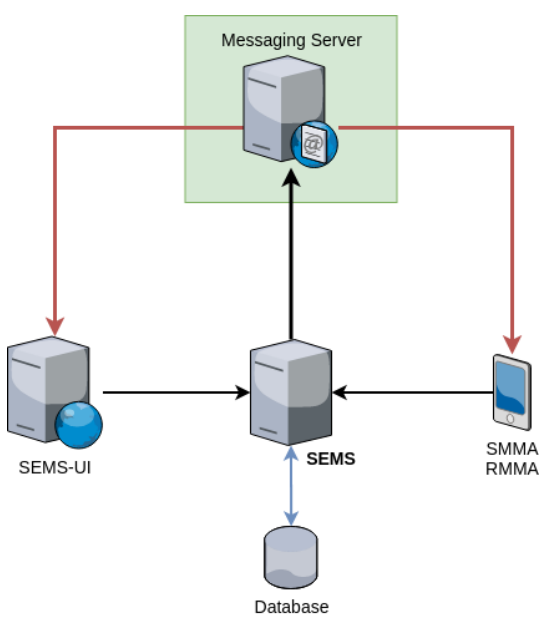

Fig. 6. The connection SEMS-RMMA-HMMA uses currently our developed Message and Alarm server (MAAS) based on Socket IO for notification and authentication.

locations. There are different approaches that are being explored, though we believe that the answer will arise from the community of the networks for the Internet of Things and we are eager in taking part in these discussions.

\section{FUTURE DIRECTIONS}

A current limitation of our research is that the survey has been only applied in a limited number of Swiss care homes, due to constraints imposed by the COVID-19 pandemic. It will be interesting to extend the study to a wider region, with more participants (both medical staff and residents).

Additionally, we would study the perception of the ecosystem beyond the originally intended use, 
i.e. extending the proposed use within a nursing home environment towards the remote monitoring of the well-being of older adults in their own accommodation.

Furthermore, the use of our proposed technology promotes independence to the elderly user, since rather than being monitored from a specific location (e.g. a smart home), they will be able to continue with their activities of daily living, where ever they are, with the safe knowledge that they are connected to the healthcare providers and relatives. They will be, effectively, operating within a "smart world" rather than a smart home.

\section{Conclusions}

Vulnerable people would benefit from adopting smart home technologies in order to remain connected and safe at their home during a time of social distancing. We are working on a system that takes into account the ethical barriers for adoption, in particular regarding privacy and security concerns, which are as important as the technological aspects of the designed system. We have shown the technical feasibility of such a system, by creating a complete ecosystem, which takes advantage of the existing mobile technologies (e.g. smartphones, tablets, smart watches) for the monitoring the wellbeing of the elderly.

The adoption of such a system would allow medical staff to better fulfil their role whilst supporting the safety and well-being of the elderly, as well as preserving their independence as they will not be confined to being in a specific location for their monitoring. By adopting our system, the elderly can continue to enjoy an independent life whilst enjoying the benefits of a safe and private supervision by the medical staff. Preserving this independence for as long as possible is one of the key aspects of well-being, and an important aspect of life that we can aspire for our elders to continue enjoying.

\section{ACKNOWLEDGMENTS}

The authors would like to thank former students at the department of Computer Science for their work in various aspects of this project: Sylvain Renaud, Rafic Galli, Nicolas Zurbuchen, Aulelien Etiennne, Willian Greppi, and Armando Koljaj.

\section{REFERENCES}

[1] R. Armitage and L. B. Nellums, "Covid-19 and the consequences of isolating the elderly," The Lancet Public Health, vol. 5, no. 5, p. e256, 2020.

[2] World-Health-Organisation. (2020) 2019 novel coronavirus (2019-ncov): Strategic preparedness and response plan. [Online]. Available: https://www.who.int/docs/defaultsource/coronaviruse/srp-04022020.pdf

[3] S. Amin, "The psychology of coronavirus fear: Are healthcare professionals suffering from corona-phobia?" International Journal of Healthcare Management, pp. 1-8, 2020. [Online]. Available: https:/ / doi.org/10.1080/20479700.2020.1765119

[4] P. Pirzada, A. Wilde, and D. Harris-Birtill, "Smart homes for elderly to promote their health and wellbeing," September 2019. [Online]. Available: https://researchrepository.st-andrews.ac.uk/handle/10023/18476

[5] P. Pirzada, N. White, and A. Wilde, "Sensors in smart homes for independent living of the elderly," in $5^{\text {th }}$ International Multi-Topic ICT Conference (IMTIC), IEEE. IEEE, April 2018, pp. 1-8.

[6] L. Yu, W. M. Chan, Y. Zhao, and K.-L. Tsui, "Personalized health monitoring system of elderly wellness at the community level in hong kong," IEEE Access, vol. 6, pp. 35558-35567, 2018.

[7] P. Pirzada, A. Wilde, G. H. Doherty, and D. Harris-Birtill, "Ethics and acceptance of smart homes for the elderly," (currently under review)., pp. 1-12, 2020.

[8] N. Zurbuchen, P. Bruegger, and A. Wilde, "A comparison of machine learning algorithms for fall detection using wearable sensors," in 2020 International Conference on Artificial Intelligence in Information and Communication (ICAIIC). Japan: IEEE, 2020, pp. 427-431.

[9] L. Z. Rubenstein, "Falls in older people: epidemiology, risk factors and strategies for prevention," Age and Ageing, vol. 35, no. suppl_2, pp. ii37-ii41, 09 2006. [Online]. Available: https://doi.org/10.1093/ageing/afl084

[10] World-Health-Organisation. (2008) Who global report on falls prevention in older age. [Online]. Available: https:/ / extranet.who.int/agefriendlyworld/wpcontent/uploads/2014/06/WHo-Global-report-on-fallsprevention-in-older-age.pdf

[11] S. Sadigh, A. Reimers, R. Andersson, and L. Laflamme, "Falls and Fall-Related Injuries Among the Elderly: A Survey of Residential-Care Facilities in a Swedish Municipality," Journal of Community Health, no. 29, pp. 129-140, 2004.

[12] D. Wild, U. S. Nayak, and B. Isaacs, "How dangerous are falls in old people at home?" The British Medical Journal, vol. 282, pp. 266-268, 1981.

[13] P. Rashidi and A. Mihailidis, "A Survey on AmbientAssisted Living Tools for Older Adults," IEEE Journal of Biomedical and Health Informatics, vol. 17, pp. 579-590, 2013.

[14] H. Hawley-Hague, E. Boulton, A. Hall, K. Pfeiffer, and C. Todd, "Older adults' perceptions of technologies aimed at falls prevention, detection or monitoring: A systematic review," International Journal of Medical Informatics, vol. 83, pp. 416-426, 2014. 